\section{A randomised controlled trial evaluating the use of enteral nutritional supplements postoperatively in malnourished surgical patients}

\author{
A H Beattie, A T Prach, J P Baxter, C R Pennington
}

Protein energy malnutrition is still a common problem in hospital patients. Early studies reported a prevalence of malnutrition of $40-50 \%$ in surgical and medical hospital patients. ${ }^{1}$ A subsequent study demonstrated that up to $40 \%$ of patients were malnourished on admission to hospital. The majority of these patients experienced nutritional depletion during the course of their admission, and nutritional depletion during hospital stay was more common and more severe in those patients who were already depleted at the time of admission. ${ }^{2}$ There are limited data on nutritional status following discharge from hospital, and few studies have addressed the prevalence of nutritional depletion in the community setting.

Malnutrition is associated with tissue wasting and impaired organ function which leads to increased morbidity and longer periods of hospitalisation. ${ }^{34}$ Impaired immune function contributes to increased infection risk, ${ }^{5}$ and muscle function has been shown to be adversely affected by nutritional depletion. ${ }^{6}$ Loss of muscle power and increased muscle fatigability will delay mobilisation and affect respiratory and cardiac function. ${ }^{78}$ Malnourished patients are thus at risk of cardiorespiratory impairment, and chest and wound infections.

Nutritional support leads to improved nutritional status ${ }^{9}$ and clinical outcome ${ }^{10}$ in severely depleted patients. Studies of nutritional repletion in malnourished patients with fractured neck of femur, ${ }^{11}{ }^{12}$ patients in care on an elderly ward, ${ }^{13}$ and studies of preoperative ${ }^{14}$ and postoperative nutritional support ${ }^{15} 16$ have demonstrated reduced morbidity and/or reduced length of hospital stay. Information on repletion after discharge is limited, particularly in the mildly depleted patient.

Patients who undergo major surgery are at risk of malnutrition due to starvation, the stress of surgery, and the subsequent increase in metabolic rate. Gastrointestinal surgery can create additional problems as it often directly affects and limits dietary intake postoperatively and these effects continue after discharge. One study in the community reported that $10 \%$ of patients who had undergone major surgery within six weeks of being surveyed, of whom $20 \%$ had undergone gastrointestinal surgery, were malnourished. ${ }^{17}$

Abbreviations used in this paper: QOL, quality of life; BMI, body mass index; MAC, mid arm circumference; MAMC, mid arm muscle circumference; TSF, triceps skinfold thickness. 


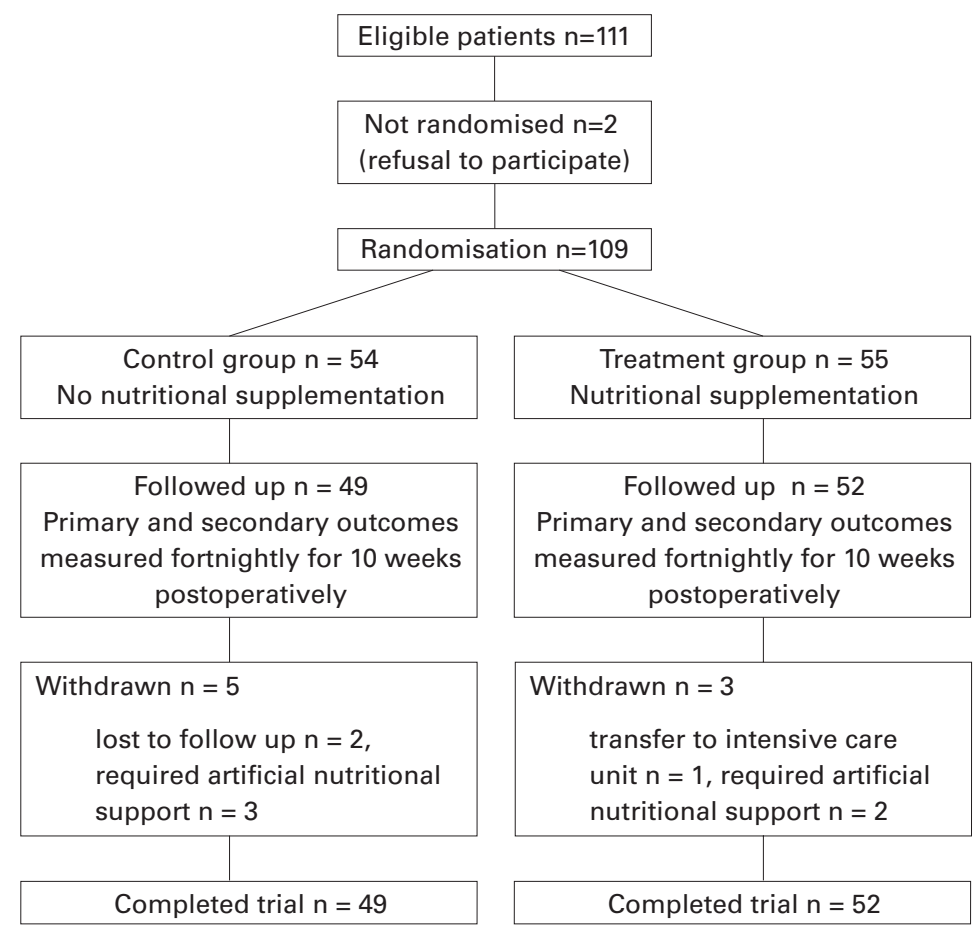

Figure 1 Flow chart describing the progress of patients through the clinical trial.

Evidence suggests that artificial nutritional support in malnourished patients reduces the costs of health care resources by reducing length of stay, morbidity, and improving quality of life. ${ }^{18}$

This study was designed to investigate postoperative malnutrition to determine the extent of nutritional depletion in hospital and in the community after gastrointestinal and vascular surgery. We also investigated the effect of postoperative oral supplementation on nutritional status, morbidity, and quality of life in malnourished surgical patients.

\section{Methods}

The study was conducted with the approval of Tayside Committee on Medical Research Ethics and all patients gave informed consent. This was a prospective, randomised, controlled trial conducted over 18 months. Patients between the ages of 18 and 80 years admitted to Ninewells Hospital and Medical School for elective gastrointestinal or vascular surgery were considered for enrolment. During the 18 month period, 2486 patients were admitted to the wards in which the study was undertaken, of which 450 were screened. There were no exclusions during the screening process. $\mathrm{Pa}-$ tients were assessed on admission to hospital and again on initiation of the oral diet postoperatively. Entry was determined by the presence of malnutrition defined by anthropometric criteria (see below) on admission or on resumption of the oral diet by the eighth postoperative day, and/or a weight loss of $5 \%$ or more from admission until oral intake was resumed by the eighth postoperative day. Patients who required parenteral nutrition and those who were pregnant or lactating were excluded. Also excluded were patients with terminal diseases and those with decompensated liver or renal disease.

On initiation of the oral diet (this will now be referred to as the point of inclusion) patients who fulfilled the study criteria were randomised to a control (CG) or treatment (TG) group. Randomisation was performed using a computer generated table of random numbers. The control group continued with routine nutritional management. Group TG was provided with an oral dietary supplement (Ensure Plus, Ross Laboratories, UK) which provided $1.5 \mathrm{kcal}$ and $0.06 \mathrm{~g} / \mathrm{ml}$ protein. Patients were encouraged to aim to consume $400 \mathrm{ml}$ of the supplements in small, frequent amounts in between meals to increase nutrient intake. All patients were then assessed by means of a home visit every two weeks postoperatively for 10 weeks. The assessments in this trial were not made blind to treatment. To reduce the amount of bias of this approach, standard equipment, assessments, and techniques were undertaken throughout. A study of 100 patients was planned as previous publications investigating the influence of oral supplements/ nutritional support showed significant effects with similar numbers of patients. The length of the trial was 18 months as this was the duration of the funding.

Patients were assessed to determine their nutritional status and skeletal muscle function using handgrip dynamometry and clinical outcome. These measurements were undertaken on admission to hospital, on inclusion, and every two weeks for the duration of the study. Quality of life (QOL) was measured on inclusion and at the end of the study.

Nutritional status was documented by anthropometry. Weight was measured on electronic scales. Height was measured using a stadiometer or knee caliper and body mass index (BMI) calculated $(\mathrm{BMI}=$ weight $(\mathrm{kg}) /$ height $\left.(\mathrm{m})^{2}\right)$. Mid arm circumference (MAC) $(\mathrm{cm})$ was measured using a non-stretch plastic tape measure and triceps skinfold thickness (TSF) $(\mathrm{mm})$ using Harpenden skinfold calipers. Both MAC and TSF were measured on the non-dominant arm using accepted methods. ${ }^{19}$ MAC and TSF were then used to calculate mid arm muscle circumference (MAMC) according to the formula: MAMC $(\mathrm{cm})=$ MAC $(\mathrm{cm})-\mathrm{TSF}(\mathrm{mm}) \times 0.3142$. For the purpose of this study malnutrition on admission to hospital, or at the point of inclusion, was defined as BMI $\leqslant 20 \mathrm{~kg} / \mathrm{m}^{2}$ and TSF or MAMC $\leqslant 15$ th percentile and/or weight loss $\geqslant 5 \%$ from admission to hospital to the point of inclusion. Patients with a BMI of $\leqslant 18$ and $\leqslant 16 \mathrm{~kg} / \mathrm{m}^{2}$ with anthropometric measurements below the 5th percentile were considered to have moderate and severe malnutrition, respectively. A BMI of $\geqslant 25$ was defined as overweight.

Handgrip strength was measured using a Harpenden handgrip dynamometer. Patients were asked to grip the dynamometer with the non-dominant hand three times and the highest reading was taken (kg). Clinical outcome was assessed by documentation of the incidence of wound and chest infections. The definition of a wound or chest infection was 
Table 1 Diagnoses of subjects in the control and treatment groups

\begin{tabular}{lcc}
\hline Diagnosis & Control & Treatment \\
\hline Ca oesophagus & 4 & 5 \\
Ca stomach & 5 & 8 \\
Ca colon & 6 & 4 \\
Ca rectum & 3 & 3 \\
Ca pancreas & 1 & 0 \\
Ca gallbladder & 1 & 0 \\
Inflammatory bowel disease & 4 & 10 \\
Diverticulitis & 3 & 1 \\
Small bowel lymphoma & 1 & 0 \\
Gastric/peptic ulcer & 2 & 1 \\
Small bowel stricture & 2 & 1 \\
Dumping syndrome & 1 & 0 \\
Vascular disease & 3 & 6 \\
Small bowel adhesions & 4 & 4 \\
GI obstruction & 5 & 3 \\
Prolapsed ileostomy & 2 & 1 \\
Gastric reflux & 1 & 0 \\
Reversal Hartmann's & 1 & 1 \\
Myelofibrosis & 0 & 1 \\
Obstructive jaundice & 0 & 2 \\
Colitic perforation & 0 & 1 \\
Total & 49 & 52 \\
\hline
\end{tabular}

Ca, Cancer; GI, gastrointestinal.

that diagnosed and treated by medical staff during the hospital stay, or a general practitioner or district nurse following discharge into the community. Requirements for antibiotic prescriptions were also recorded.

QOL was assessed using the well validated UK SF-36 questionnaire. ${ }^{20}$ This was completed by each patient on inclusion and at completion of the study. The UK SF-36 QOL questionnaire is a self administered questionnaire containing 36 items which takes about five minutes to complete. It measures health on eight multidimensions, covering functional status, well being, and overall evaluation of health.

The following statistical analysis methods were used. Data obtained on weight, MAC, TSF, MAMC, and grip strength were analysed using one way analysis of variance (ANOVA) to determine linear trend between time points. Two way ANOVA analysis was used to examine the difference between the CG and TG groups and between time points. The Mann Whitney $\mathrm{U}$ test assessed the change between QOL questionnaires completed at the initial and final assessments. Differences in clinical outcome in both groups were compared using the $\chi^{2}$ test.

\section{Results}

PATIENTS

Four hundred and fifty patients were assessed of which 111 met the criteria for entry. Two patients declined to participate. The resulting 109 patients were recruited into the study on

Table 2 Nutritional status of patients on admission to hospital and postoperatively on inclusion into the study

\begin{tabular}{|c|c|c|c|c|c|}
\hline & \multirow[b]{2}{*}{$B M I\left(\mathrm{~kg} / \mathrm{m}^{2}\right)$} & \multicolumn{2}{|c|}{ On admission } & \multicolumn{2}{|c|}{ On inclusion } \\
\hline & & Control & Treatment & Control & Treatment \\
\hline Any malnutrition & $\leqslant 20$ & 20 & 20 & 30 & 35 \\
\hline Severe & $\leqslant 16$ & 1 & 0 & 2 & 1 \\
\hline Moderate & $\leqslant 18$ & 4 & 2 & 9 & 5 \\
\hline Mild & $\leqslant 20$ & 15 & 18 & 19 & 29 \\
\hline Normal & $20-25$ & 24 & 28 & $16^{\star}$ & $13^{\star}$ \\
\hline Overweight & $\geqslant 25$ & 5 & 4 & $3^{\star}$ & $4^{\star}$ \\
\hline
\end{tabular}

* These patients were recruited due to $\geqslant 5 \%$ weight loss in the period between admission and inclusion into the study
Table 3 Surgeries performed in subjects in the control and treatment groups

\begin{tabular}{lll}
\hline Surgery & Control & Treatment \\
\hline Gastrectomy & 7 & 9 \\
Total colectomy & 1 & 0 \\
Left hemicolectomy & 3 & 3 \\
Right hemicolectomy & 3 & 5 \\
Sigmoid colectomy & 3 & 3 \\
Ileoanal pouch formation & 0 & 1 \\
Abdominoperineal resection & 6 & 3 \\
Small bowel resection & 3 & 6 \\
Roux-en-Y diversion & 1 & 0 \\
Oesophagectomy & 4 & 5 \\
Amputation toe & 0 & 1 \\
Laparotomy and resection & 4 & 4 \\
Resection recto-sigmoid anterior & 2 & 0 \\
$\quad$ junction & & \\
Ileostomy reconstruction & 2 & 1 \\
Vascular surgery & 3 & 5 \\
Anterior resection & 1 & 2 \\
Choleduodenostomy & 1 & 0 \\
Fundoplication & 1 & 0 \\
Open cholecystectomy & 1 & 1 \\
Reversal Hartmann's & 0 & 1 \\
Splenectomy & 0 & 1 \\
Resection of jejuno-colic & 1 & 0 \\
$\quad$ anastomosis & & \\
Pancreatoduodenectomy & 1 & 0 \\
Hartmann's & 0 & 1 \\
Ileojejunal anastomosis & 1 & 0 \\
\hline & &
\end{tabular}

resumption of oral intake postoperatively and randomised to one of two groups (54 in the CG group and 55 in the TG group). Eight subjects were withdrawn (five from the control group and three from the TG group for the following reasons). Two patients from the CG group could not be contacted following discharge from hospital, one patient from the TG group was transferred to intensive care with a chest infection after resumption of an oral diet, one patient in the control group and one in the TG group were commenced on enteral tube feeding, and three patients (two from group CG and one from group TG) required parenteral feeding due to fistula development and chronic malabsorption. The resulting 101 patients completed the study. The flow diagram (fig 1) shows the progress of the patients through the trial.

Patient diagnoses are listed in table 1 . The distribution of benign and malignant disease was similar between groups. In the control group there were 28 patients with benign disease and 21 with malignant disease; corresponding values in group TG were 32 and 20, respectively. The mean (SD) age of the subjects was $62.4(10.9)$ years in the control group and 54.4 (19.4) years in the treatment group. The mean age of subjects in the treatment group was younger by less than 10 years $(p<0.05)$ but

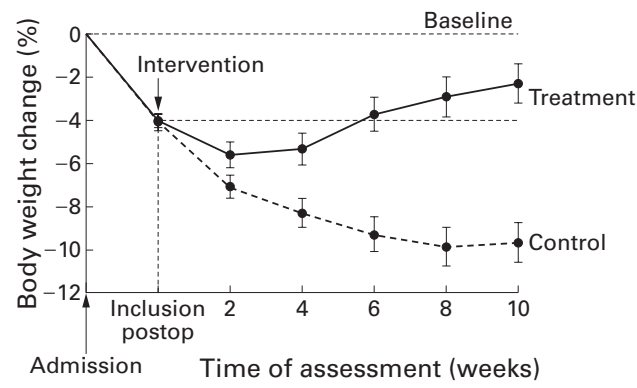

Figure 2 Percentage change in body weight in the control and treatment groups on admission to hospital, at inclusion in the study, and then at two weekly intervals for 10 weeks. 
Table 4 Changes in nutritional variables at each assessment point from time of admission

\begin{tabular}{|c|c|c|c|c|c|c|c|c|c|c|c|}
\hline & \multirow[b]{2}{*}{ Inclusion } & \multirow[b]{2}{*}{2 weeks } & \multirow[b]{2}{*}{4 weeks } & \multirow[b]{2}{*}{6 weeks } & \multirow[b]{2}{*}{8 weeks } & \multirow[b]{2}{*}{10 weeks } & \multicolumn{2}{|c|}{${ }^{\star}$ Linear trend } & \multicolumn{3}{|c|}{ **Difference } \\
\hline & & & & & & & $F$ & $p$ & & $F$ & $p$ \\
\hline \multicolumn{12}{|c|}{ Weight loss (kg) } \\
\hline Control & $2.28(1.28)$ & $4.21(2.44)$ & $5.13(3.23)$ & $5.68(3.90)$ & $5.96(4.21)$ & $5.86(4.33)$ & 33.6 & $<0.001$ & (1) & 71.53 & $<0.001$ \\
\hline Treatment & $2.31(1.36)$ & $3.40(2.94)$ & $3.40(3.26)$ & $2.48(3.58)$ & $1.89(4.27)$ & $1.53(4.23)$ & 5.48 & 0.02 & (2) & 4.34 & 0.001 \\
\hline \multicolumn{12}{|c|}{$\begin{array}{l}\text { Decrease in TSF } \\
(\mathrm{mm})\end{array}$} \\
\hline Control & $0.10(0.32)$ & $0.32(0.90)$ & $0.51(1.19)$ & $0.72(1.32)$ & $0.80(0.42)$ & $0.82(1.41)$ & 3.09 & 0.01 & (1) & 22.01 & $<0.001$ \\
\hline Treatment & $0.19(0.68)$ & $0.11(0.94)$ & $0.26(0.77)$ & $0.07(0.82)$ & $0.02(0.90)$ & $0.16(1.73)$ & 0.42 & NS & (2) & 1.44 & NS \\
\hline \multicolumn{12}{|c|}{$\begin{array}{l}\text { Decrease in MAMC } \\
(\mathrm{cm})\end{array}$} \\
\hline Control & $0.56(1.30)$ & $1.01(1.80)$ & $1.27(1.91)$ & $1.29(1.94)$ & $1.37(1.90)$ & $1.28(1.73)$ & 4.88 & $<0.03$ & (1) & 17.16 & $<0.001$ \\
\hline Treatment & $0.55(0.75)$ & $0.86(0.94)$ & $0.81(0.82)$ & $0.71(0.83)$ & $0.61(0.92)$ & $0.42(1.01)$ & 2.10 & NS & (2) & 1.64 & NS \\
\hline \multicolumn{12}{|c|}{$\begin{array}{l}\text { Decrease in grip } \\
\text { strength }\left(\mathrm{kg} / \mathrm{m}^{2}\right)\end{array}$} \\
\hline Control & $1.56(1.82)$ & $2.51(3.13)$ & $2.45(2.99)$ & $2.16(2.41)$ & $2.10(2.35)$ & $1.93(2.21)$ & 0.01 & NS & (1) & 13.58 & $<0.001$ \\
\hline Treatment & $1.73(1.87)$ & $1.82(1.92)$ & $1.95(2.80)$ & $1.17(1.64)$ & $1.04(2.00)$ & $0.82(2.10)$ & 9.94 & $<0.005$ & (2) & 2.12 & NS \\
\hline
\end{tabular}

Values are mean (SD).

${ }^{\star}$ One way ANOVA for differences between time points. ${ }^{\star}$ Two way ANOVA: (1) difference between control and treatment groups; (2) difference between time points.

there was no significant difference in the numbers of patients in the control and treatment groups $<65$ years $(23 / 49 v 29 / 52)$ and $>65$ years (26/49 $v 23 / 52)$. There were 33 males and 16 females in the control group and 27 males and 25 females in the treatment group. The mean (SD) length of time from surgery to inclusion in the study was similar between the two groups (6.5 (1.4) days in the control group and 6.5 (1.6) days in the treatment group).

\section{NUTRITIONAL STATUS}

The nutritional status of patients on admission and on inclusion in the study, as defined by anthropometry, is given in table 2. Sixty five of $101(64 \%)$ patients were recruited on the basis of anthropometric criteria alone; 36/101 (36\%) were recruited on the basis of $\geqslant 5 \%$ weight loss between the time of admission and inclusion in the study. Surgeries performed are shown in table 3

The percentage change in body weight between the two groups from admission to hospital, inclusion in the study, and then at two weekly intervals for 10 weeks is shown in fig 2 . Table 4 shows the changes in weight, TSF, MAMC, and grip strength. There was no significant difference between the two groups in weight loss from admission through to the operative period until the point of intervention postoperatively. Thereafter the CG group lost more weight overall than the TG group, with a maximum loss of 5.96 compared with $3.40 \mathrm{~kg}$. In terms of percentage body weight, this is the equivalent of $9.8 \%$ versus $5.6 \%$. The TG group showed evidence of improving nutritional status four weeks after surgery. The CG group demonstrated progressive weight loss for eight weeks following surgery. The anthropometric measurements TSF and MAMC showed similar significant differences as weight change in the CG and TG groups, indicating relative body protein and body fat depletion $(\mathrm{p}<0.001)$.

The pattern of weight change was similar in patients with benign and malignant disease (table 5). Although the numbers were too small for meaningful statistical analysis, the treatment group lost less weight and regained weight earlier than the control group in both the benign and malignant categories.

There was progressive reduction in grip strength in both the CG and TG groups up to four weeks after surgery but the reduction in the TG group was less marked with significantly improved values in grip strength apparent at 10 weeks in comparison with the CG group $\left(-0.82 v-1.93 \mathrm{~kg} / \mathrm{m}^{2} ; \mathrm{p}<0.001\right)$.

\section{NUTRITIONAL SUPPLEMENTS}

Compliance was monitored by asking patients how much of the nutritional supplements were consumed; in practice the majority of patients took 200-400 $\mathrm{ml}$ daily.

\section{CLINICAL OUTCOME}

Table 6 shows the incidence of complications and relative risk. We found that the risk of chest and wound infections was lower in the TG group than in the CG group: 15/49 subjects in the CG group required one or more prescriptions for antibiotics compared with only $7 / 52$ in the TG group $(\mathrm{p}<0.05)$ but the adjusted analysis for age and sex did not show statistical significance. There was no difference in length of hospital stay between the two groups (20.6 (15.0) days in the CG and 18.4 (9.9) days in the TG group). There were no deaths during the study.

Table 5 Weight loss $(\mathrm{kg})$ in patients with benign and malignant disease at each assessment point from time of admission

\begin{tabular}{lllllll}
\hline & Inclusion & 2 weeks & 4 weeks & 6 weeks & 8 weeks & 10 weeks \\
\hline Benign & & & & & & \\
$\quad$ Control $(\mathrm{n}=28)$ & $2.03(1.33)$ & $3.98(2.75)$ & $4.53(3.26)$ & $5.01(3.74)$ & $5.34(4.17)$ & $5.22(4.42)$ \\
$\quad \begin{array}{l}\text { Treatment }(\mathrm{n}=32) \\
\text { Malignant }\end{array}$ & $2.34(1.30)$ & $3.20(2.64)$ & $2.98(3.27)$ & $1.98(3.73)$ & $1.26(4.49)$ & $0.98(4.37)$ \\
$\quad$ Control $(\mathrm{n}=21)$ & $2.60(1.17)$ & $4.51(1.97)$ & $5.93(3.10)$ & $6.28(4.12)$ & $6.51(4.31)$ & $6.44(4.23)$ \\
$\quad$ Treatment $(\mathrm{n}=20)$ & $2.25(1.49)$ & $3.71(3.40)$ & $4.1(3.21)$ & $3.29(3.25)$ & $2.80(3.85)$ & $2.25(3.95)$ \\
\hline
\end{tabular}

Values are mean $(\mathrm{SD})$ 
Table 6 Incidence of complications in the control and treatment groups

\begin{tabular}{lllllllll}
\hline & $\begin{array}{l}\text { Control } \\
(n=49)\end{array}$ & $\begin{array}{l}\text { Treatment } \\
(n=52)\end{array}$ & $R R$ & $95 \% C I$ & $R R^{\star}$ & $95 \% C I$ & $R R^{\star *}$ & $95 \% C I$ \\
\hline Infection & & & & & & & & \\
$\quad$ Chest & 6 & 2 & 0.31 & $0.07-1.48$ & 0.34 & $0.07-1.73$ & 0.37 & $0.07-1.93$ \\
Wound & 7 & 4 & 0.53 & $0.17-1.73$ & 0.62 & $0.18-2.14$ & 0.60 & $0.17-2.08$ \\
Antibiotics & 15 & 7 & 0.43 & $0.19-0.97 \dagger$ & 0.49 & $0.20-1.22$ & 0.50 & $0.20-1.25$ \\
\hline
\end{tabular}

${ }^{\star}$ Adjusted for age (continuous); ${ }^{\star \star}$ adjusted for age and sex.

$+\mathrm{p}<0.05$.

Table 7 Quality of life measurement for physical and mental component summary scores

\begin{tabular}{|c|c|c|c|c|c|c|}
\hline & \multicolumn{3}{|l|}{ Physical score } & \multicolumn{3}{|l|}{ Mental score } \\
\hline & $\begin{array}{l}\text { Treatment } \\
(n=52)\end{array}$ & Control $(n=49)$ & $\begin{array}{l}\text { All patients } \\
(n=101)\end{array}$ & $\begin{array}{l}\text { Treatment } \\
(n=52)\end{array}$ & $\begin{array}{l}\text { Control } \\
(n=49)\end{array}$ & $\begin{array}{l}\text { All patients } \\
(n=101)\end{array}$ \\
\hline Initial assessment (A) & $-13.8(43.4)$ & $-18.0(33.5)$ & $-15.8(38.9)$ & $4.8(43.6)$ & $6.3(35.8)$ & $5.5(39.9)$ \\
\hline Final assessment (B) & $7.3(47.3)$ & $-13.9(38.6)$ & $-2.7(44.5)$ & $20.8(46.1)$ & $7.2(39.1)$ & $14.4(43.3)$ \\
\hline Change $(\mathrm{B}-\mathrm{A})$ & $21.1(18.6)^{\star \star \star}$ & $4.1(17.3)^{\star \star \star}$ & $13.1(19.8)$ & $16.0(18.7)^{\star \star \star}$ & $0.9(16.9)^{\star \star \star}$ & $8.9(19.3)$ \\
\hline
\end{tabular}

Values are mean $(\mathrm{SD})$.

$\star \star \star \mathrm{p}<0.001$.

QUALITY OF LIFE

The UK SF-36 health status questionnaire can be divided into two summary scores in relation to physical and mental health (table 7). The treatment group showed statistically significant improvements in physical and mental health compared with the control group $(\mathrm{p}<0.001)$.

\section{Discussion}

The study population represents approximately $24 \%$ of patients who were screened. Thus $9 \%$ of the total population screened were entered on the basis of admission anthropometric criteria, a value lower than a previous study undertaken in this hospital examining the incidence of malnutrition. ${ }^{2}$ The lack of detail relating to all patients admitted during this period is accepted as a limitation in the interpretation of the significance of the findings. However, evidence suggests that nutritional impairment to the degree that determined entry to this study is common. The high incidence of nutritional depletion on admission to hospital has been well documented. The change in nutritional status during hospital admission has been studied less frequently. There are limited data on changes in nutritional status after surgery and following discharge from hospital. It is therefore not clear if nutritional supplementation can be justified following discharge from hospital.

We found that without nutritional intervention patients in the CG group progressively lost weight up to eight weeks after surgery with a mean loss of $9.8 \%$ body weight, and $24 / 49$ patients in the CG group lost $\geqslant 10 \%$ body weight. Administration of oral dietary supplements in the TG group resulted in a mean loss of only $5.6 \%$ body weight ( $11 / 52$ patients in the TG lost $\geqslant 10 \%$ body weight) and repletion was evident four weeks after surgery. Guidelines on nutritional support indicate that a value of $10 \%$ weight loss is clinically significant and active nutritional support is indicated. ${ }^{21}$

Both the TG and CG groups displayed corresponding reductions in MAMC and TSF during the operative period. These results are in agreement with those of a previous study of dietary supplementation in patients following surgery where weight was slow to recover in the control group compared with the group receiving nutritional supplementation. ${ }^{22}$ They are also in agreement with the systematic review by Potter and colleagues who reported that most trials included in the review concluded that nutritional supplementation improves body weight and anthropometry. ${ }^{23}$

Hand grip strength in our CG and TG groups indicated skeletal muscle function was depleted progressively up to four weeks after surgery. Values in the TG group showed that skeletal muscle function was preserved to a greater extent with values returning to those close to preoperative levels by the end of the 10 week period, which was similar to the pattern seen in other nutritional variables, typically weight and MAMC. Other studies examining the effect of nutritional support and hand grip strength have shown more rapid effects of nutritional support on hand grip strength independent of changes in MAMC. ${ }^{16}$ The positive effects of nutritional support on hand grip strength have also been shown with parenteral nutrition. ${ }^{25}$ Our results may suggest that in the malnourished patient skeletal muscle function is less efficiently replenished, with recovery not becoming apparent until 10 weeks after surgery.

Systematic review of the effects of nutritional supplementation failed to show an unequivocal effect of nutritional supplements on clinical outcome. ${ }^{23}$ We report 13 infectious complications in the control group compared with six in the treatment group. The reduction is similar to that seen in other studies. ${ }^{1624}$ This trend towards a higher infection rate in the control group did not reach statistical significance. Less patients in the treatment group (7/52) required antibiotic prescriptions compared with the control group (15/49).

QOL is an important consideration in clinical outcome. Results of the UK SF-36 scores showed a significant improvement in QOL following intervention with oral supplements in the TG group compared with the control group $(p<0.001)$. This indicates that an improvement in nutritional status is associated with improvement in QOL. This contrasts with 
the findings in a previous study ${ }^{16}$ which reported no benefit of administering oral dietary supplements to patients who had undergone moderate to major gastrointestinal surgery after discharge from hospital. This could be explained by the different patient groups studied and the surgical procedures undertaken. In our study only malnourished patients were included as opposed to patients unselected by nutritional status. The authors proposed that routine nutritional supplementation is indicated when pre-existing malnutrition is present at the time of surgery or has developed as a result of surgery, as further nutritional problems can occur following discharge from hospital. Forty three per cent of patients underwent upper gastrointestinal surgery (as defined by small bowel and above) in comparison with the study of Keele and colleagues $^{16}$ in which only $15 \%$ underwent upper gastrointestinal surgery. In clinical practice patients who have undergone upper gastrointestinal surgery are more likely to experience chronic nutritional problems due to early satiety and discomfort on eating. Dietary supplements are more easily taken and tolerated than solid food and are an effective way of enhancing nutrient intake.

In our study 41/101 patients had malignant disease. We subdivided data into malignant and benign disease and examined the results for the TG and CG groups. The distribution of benign and malignant disease was similar in both groups. Patients with malignant disease in the CG and TG groups showed more profound weight loss with slower recovery in comparison with those with benign disease. Overall, regardless of disease pathology the benefits of supplementation were apparent.

The data were not analysed on an intention to treat basis as the numbers of patients who were withdrawn was small $(7 \%)$, with similar numbers from both groups (5/49 from group CG and 3/52 from group TG). We felt it reasonable to exclude these patients as they were almost immediately denied access to prescribed dietary conditions, could not be assessed, and were therefore not comparable with the rest of the subjects. Our study did not demonstrate any effect on the length of hospital stay but intervention began up to eight days after surgery and therefore no such effect was expected. The control group did not receive any placebo therapy but were visited and measured in the same way as the TG group.

There are few conclusive data on changes in nutritional status in the operative period, the nutritional status of patients postoperatively following discharge from hospital, or on the value of oral nutritional supplements in patients with different diseases/conditions living in the community. ${ }^{26}$ Our study has shown that malnourished patients who have undergone gastrointestinal and vascular surgery continue to experience nutritional problems after discharge and this has a detrimental effect on nutritional status, QOL, and clinical outcome. Nutritional intervention can prevent such marked depletion of body tissue, improve QOL, and improve clinical outcome. We therefore support the view that nutritional status should be monitored during the perioperative and postoperative periods, and that the use of oral nutritional supplements should be considered in such malnourished patients who have undergone gastrointestinal and vascular surgery.

The authors would like to acknowledge Abbott Laboratories for funding and supporting this study, Dr Chen, Ninewells Hospital for his statistical advice, and all consultant surgeons at Ninewells Hospital for access to their patients during the study.

1 Pennington CR. Disease and malnutrition in British hospitals. Proc Nutr Soc 1997; 56:393-407.

2 McWhirter JP, Pennington CR. The incidence and recognition of malnutrition in hospital. BMF 1994;308:945-8.

3 Giner M. In 1995 a correlation still exists between malnutrition and poor outcome in critically ill patients. Nutrition 1996;12:23-9.

4 Pennington C, Powell-Tuck J, Shaffer J. Review article: artificial nutritional support for improved patient care. Aliment Pharmacol Ther 1995;9:471-81.

5 Windsor JA, Hill GL. Risk factors for post operative pneumonia: the importance of protein depletion. Ann Surg 1988;17:181-5.

6 Jeejeebhoy KN. Muscle function and nutrition. Gut 1986;27:25-39

7 Arora NS, Rochester DF. Respiratory muscle strength and maximal voluntary ventilation in undernourished patients. Am Rev Respir Dis 1982;126:5-8.

8 Heymsfield SB. Cardiac abnormalities in cachectic patients before and during nutritional repletion. Am Heart $\mathcal{F} 1978$; 95:584-95.

9 McWhirter JP, Pennington CR. A comparison between oral and nasogastric supplements in malnourished patients. Nutrition 1996;12:502-6.

10 Beier-Holgersen SR, Boesby S. Influence of postoperative enteral nutrition on post surgical infections Gut 1996;39: 833-5.

11 Bastow MD, Rawlings J, Allison SP. Benefits of supplementary tube feeding after fractured neck of femur: a randomised control trial. BMF 1993;287:1589-92.

12 Delmi M, Rapin CH, Bengoa JM, Delmas PD, et al. Dietary supplementation in elderly patients with fractured neck of femur. Lancet 1990;335:1013-16.

13 Larsson J, Unosson M, Ek AC, Nilsson L, et al. Effect of dietary supplement on nutritional status and clinical outcome in 501 geriatric patients: a randomised study. Clin Nutr 1990;9:179-84.

14 Von Meyenfeld MF, Meijerink WJHJ, Rouflard MJ, et al. Perioperative nutritional support: a randomised clinical trial Clin Nutr 1992:11:180-6.

15 Askanazi J, Starker PN, Olsson C, et al. Effect of immediate post-operative nutritional support on the length of hospitalisation. Ann Surg 1986;203:236-9.

16 Keele AM, Bray M, Emery P, et al. Two phased randomised controlled clinical trial of postoperative dietary supplements in surgical patients. Gut 1997;40:393-9.

17 Edington J, Keon P, Martyn CN. Prevalence of malnutrition in patients in general practice. Clin Nutr 1996;15:60-3.

18 Robinson G, Goldstein M, Levine G. Impact of nutritional status on DRG length of stay. FPEN 1987; 11: 49-51.

19 Heymsfield SB, McManus C, Smith J, et al. Anthropometric measurement of muscle mass: revised equations for calculating bone free arm muscle area. Am f Clin Nutr 1982;36: 680-90.

20 Brazier JE, Harper R, Jones NMB, et al. Validating the SF-36 health survey questionnaire: new outcome measure for primary care. BMF 1992;305:160-4.

21 A.S.P.E.N. Board of Directors. Guidelines for the use of parenteral and enteral nutrition in adult and paediatric patients. Parenter Enteral Nutr 1993;17:(suppl 4).

patients. Parenter Enteral Nutr 1993;17:(suppl 4).
22 Jenson MB, Hessov IB. Dietary supplementation at home improves the regain of lean body mass after surgery. Nutrition 1997; 13:422-30.

23 Potter J, Langhorne P, Roberts M. Routine protein energy supplementation in adults: systematic review. BMF 1998; 317:495-501

24 Rana SK, Bray J, Menzies-Gow N, et al. Short term benefits of post operative oral dietary supplements in surgical patients. Clin Nutr 1992;11:337-44.

25 Christie PM, Hill GL. Effect of intravenous nutrition on nutrition and function in acute attacks of inflammatory bowel disease. Gastroenterology 1990;99:730-6.

26 Stratton RJ, Elia M. A critical systemic analysis of the use of oral nutritional supplements in the community. Clin Nutr 1999;18:29-84. 